\title{
VARGUS 9: Computed stimuli for schema research'
}

SELBY H. EVANS, TEXAS CHRISTIAN UNIVERSITY MARVIN R. MUE LLER, AUGUSTA COLLEGE

A method is presented for producing stimuli by sampling from a defined population in such a way that each stimulus may be regarded as a set of measurable deviations from a prototype. Procedures are described for the measurement and control of two population characteristics derived from information the ory: stimulus channel capacity and redundancy. An interval scale measure is presented for describing individual stimuli with respect to conformity to the prototype.

The need for quantitative description and control of the stimulus in perception research has become increasingly apparent. Attneave \& Arnoult (1956) have cogently argued for random sampling of stimuli from a defined population and for quantitative descriptions of form stimuli. Fitts, Weinstein, Rappaport, Anderson, \& Leonard (1956) developed their histoform stimuli in response to the same need. The histoforms had the advantage of being readily compatible with measures of technical information and redundancy and were used to generate an impressive body of research.

A more recent system, VARGUS 7 (Evans, 1964; Evans, 1967), used a computerized pattern generation method which converted segments of a Markov process into histoform patterns ready for use as stimuli. Large numbers of patterns could be efficiently generated with this system, and the theory of Markov processes allowed substantial flexibility in the manipulation of schemata, information, and redundancy. The VARGUS 7, however, was primarily oriented toward information measures. Schema theory (see Oldfield, 1954) poses some research needs which the VARGUS 7 system cannot meet. Hence a new and complementary system, VARGUS 9 , has been developed for producing histoform stimuli.

objectives.

The VARGUS 9 system retains some of the objectives of the VARGUS 7 system: computer generation of stimuli, random sampling from a defined population, and control of information and redundancy measures. In addition, the needs of schema research dictated the following new objectives: (1) The population should be made up of variants which consist of independent and measurable deviations from a well defined prototype. (2) The deviations should be produced in such a way that encoding them in the form of schema plus correction (see Oldfield, 1954) would sharply reduce information storage requirements. (3) The deviations should be produced in such a way that it is reasonable to treat them as interval scale deviations from a mean, so that interval scale measures can be applied both to stimuli and to populations.

\section{Method}

The basic program of the system, VARGUS 9, produces cards which can be printed as histoforms on an IBM 1401 with space suppress feature. Examples of the patterns are presented in Fig. 1. The number of columns in a stimulus may be fixed at any value from one through 32 . A prototype is produced by selecting for each column, an average height ranging from three to seven units. This set of average heights constitutes the prototype, as illustrated in Fig. 1. The selection of prototype heights may be at random although most experimenters will want to use some constraints to eliminate potentially familiar configurations.

Variants a re produced in a column by column operation which maps a prototype column into the corresponding variant column according to the following rule:

$$
V_{i j}=P_{i}+d_{i j} \text {, }
$$

where $V_{i j}$ is the height of the $i$ th column of variant $j$, $P_{i}$ is the height of the $i$ th column of the prototype, and $d_{i j}$ is a random variable which takes on a distinct and independent value for each pair of $i, j$ values. Furthermore, $d$ takes on five integer values ranging from +2 to

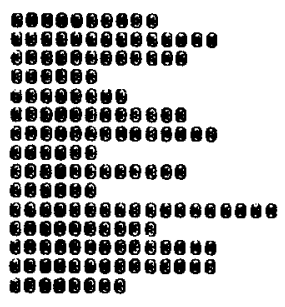
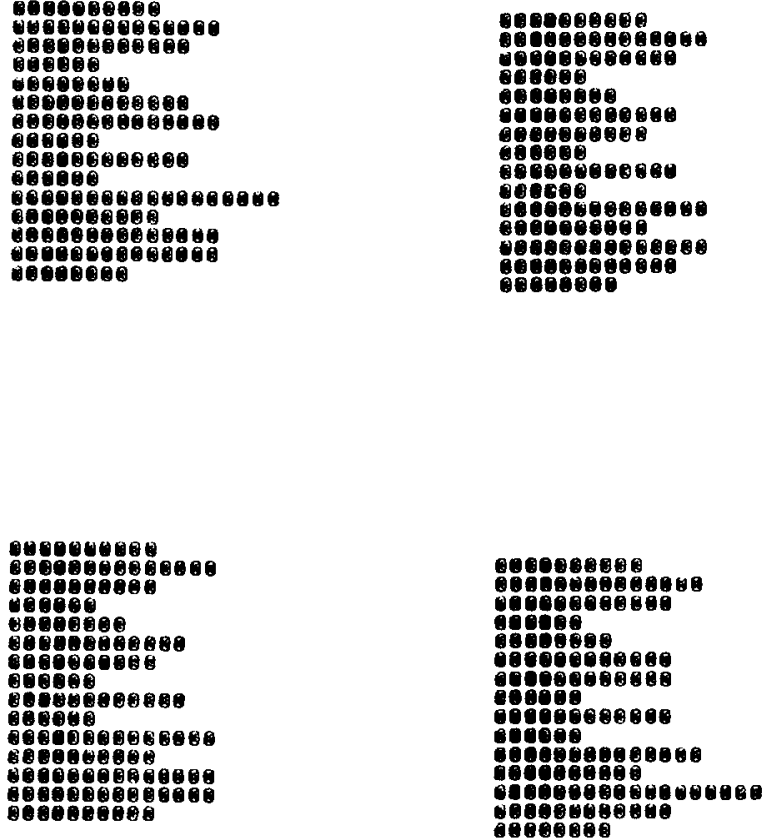

Fig. 1. Examples of VARGUS 9 stimuli. The upper left hand stimulus is a prototype. The others are variants produced at a redundancy level of $70 \%$. The stimuli were printed by an $18 M 1401$, using the V-7 WRT program (Evans, 1967). 
-2 and is unimodally and symmetrically distributed about a mean of zero. The distribution of $d$ is the same for all columns. Within the above restrictions, the particular probabilities are specified by the user and varied to manipulate the average extent to which populations conform to the prototype.

\section{Information measures.}

In the application of information measures to pattern research, confusion can be avoided by explicit and consistent use of a single set of identification statements relating the experimental situation to the information model. While other identifications are possible, we regard the following set as most generally applicable. Stimuli are identified as signals in a channel. The $E$ is identified as a message source and encoder producing the stimuli-signals. Depending on the task, $S$ is identified with the decoder or with part of the channel. Under these identifications, three quantities of interest can be obtained from the stimulus generation procedure: maximum channel capacity $\left(\mathrm{H}_{\max }\right)$, actual channel capacity $\left(\mathrm{H}_{\mathrm{a}}\right)$, and constraint redundancy $\left(\mathrm{R}_{\mathrm{c}}\right)$.

The channel capacities may be readily calculated in bits per column. Since $\mathrm{H}_{\max }$ corresponds to strictly random selection among nine column heights, $\mathrm{H}_{\max }=$ 3.170 bits per column. $\mathrm{H}_{\mathrm{a}}$ may be obtained with Shannon's formula (Shannon \& Weaver, 1949) from the probability distribution of $d$. In the VARGUS 9 system, all columns have the same uncertainty; thus channel capacity in bits per stimulus may be obtained by multiplying the above quantities by the number of columns in a stimulus. $R_{c}$ may be obtained with another of Shannon's formulae from $H_{\max }$ and $\mathrm{H}_{\mathrm{a}}$. Because $\mathbf{R}_{\mathrm{c}}$ is a ratio, it does not depend on the number of columns in a stimulus.

An experimenter may wish not merely to calculate, but to choose a particular value of $R_{c}$ or $\mathrm{H}_{a}$; for example, he might want sets stimuli which are $30 \%, 50 \%$ or $70 \%$ redundant. Shannon's definition of $R_{C}$ is easily solved to obtain the required $\mathrm{H}_{\mathrm{a}}$ for a chosen value of $R_{c}$. The problem is thus reduced to finding a probability distribution for $d$ which gives a specified value for $\mathrm{H}_{a}$. $\mathrm{H}_{a}$, however, is not sufficient to determine a unique distribution. In our present work, we have used the following special restrictions on the distribution of $\mathrm{d}$ : $\mathrm{p}(0)=\mathrm{p}_{\max } ; \mathrm{p}(1)=\mathrm{p}(-1)=1 / 3\left(1-\mathrm{p}_{\max }\right) ; \mathrm{p}(2)=\mathrm{p}(-2)=1 / 6$ $\left(1-p_{\max }\right)$. Given $H_{a}$ and these restrictions, Shannon's definition can be reduced to an equation in one unknown, $p_{\max }$. An explicit solution for $\mathrm{p}_{\max }$ is not readily obtained, but a simple program can produce a table giving $\mathrm{H}_{\mathrm{a}}$ as a function of $\mathrm{p}_{\text {max }}$. With this table, one can select the necessary $p_{\max }$ for any desired $\mathrm{H}_{\mathrm{a}}$. The $p_{\max }$ values most likely to be of interest to experimenters are those corresponding to the following redundancy levels: $30 \%, \mathrm{p}_{\max }=.31 ; 40 \%, \mathrm{p}_{\max }=.53 ; 50 \%$, $\mathrm{p}_{\max }=.66 ; 60 \%, \mathrm{p}_{\max }=.77,70 \%, \mathrm{p}_{\max }=.84$.

New features for schema research.

Objective 1 is evidently satisfied by the above system.
Furthermore, the possible column heights range from one to nine, but in any given column the heights is never more than two units from the prototype height. Therefore, encoding each column height as deviation from the prototype height reduces the number of alternatives from nine to five, satisfying objective 2 .

For any given column the population distribution of column heights resembles many naturally occurring distributions of interval scale variables (objective 3 ), in that small deviations from the mean are more probable than large deviations. Given that an interval scale is appropriate, a pattern variance (PV) can be obtained for each variant as it is generated. This quantity is merely the sample variance for the set of $d$ values used in that stimulus. More formally,

$$
P V_{j}=1 / r \quad \sum_{i=1}^{r} d_{i j}^{2},
$$

where $r$ is the number of columns in each variant. $P V$ describes each stimulus with respect to the extent to which it conforms to the prototype. A corresponding population measure is the variance of $d$.

\section{Applications.}

This system was developed to support research in schema theory. It is currently in use in studies of learning and performance as a function of redundancy and in a study of the usefulness of PV as a predictor of performance with individual patterns. PV should also be of interest as an index of similarity between individual variant and the prototype. Another measure, analogous to PV, could be obtained for any pair of patterns by summing the squared column by column differences. Such a measure would be of interest as a possible measure of similarity between pairs of patterns. A corresponding measure could be obtained for pairs of schemata, perhaps as an indicator of the ease with which Ss would learn to discriminate between schemata.

This list of applications is intended to be suggestive rather than exhaustive.

\section{References}

Attneave, F., \& Amoult, M. D. The quantitative study of shape and pattern perception. Psychol. Bull., 1956, 53, 452-47i.

Evans, S. H. A model for perceptual category formation. Unpublished doctoral dissertation, Texas Christian University, 1964.

Evans, S. H. VARGUS 7: Computed patterns from Markov processes. Behav. Sci., 1967, in press.

Fitts, P. M., Weinstein, M., Rappaport, M., Anderson, Nancy, \& Leonard, J. A. Stimulus correlates of visual pattern recognition: A probability approach. J. exp. Psychol., 1956, 51, 1-11.

oldfield, R. C. Memory mechanisms and the theory of schemata. British J. Psychol., 1954, 45, 14-23.

Shannon, C. E., \& Weaver, W. The mathematical theory of communication. Urbana, Illinois: The University of Illinois Press, 1949.

\section{Note}

1. This research was in part supported by research grant $\mathrm{MH}-12223-$ 01 from the National Institutes of Health. Additional support was provided by the Texas Christian University Computer Center. The programs described may be obtained from the T.C.U. Computer Center. 